\title{
Some Recent Developments in the Decoherent Histories Approach to Quantum Theory
}

\author{
Jonathan Halliwell \\ Blackett Laboratory, Imperial College, London, SW7 $2 B Z$
}

\begin{abstract}
A brief introduction to the decoherent histories approach to quantum theory is given, with emphasis on its role in the discussion of the emergence of classicality from quantum theory. Some applications are discussed, including quantum-classical couplings, the relationship of the histories approach to quantum state diffusion, and the application of the histories approach to situations involving time in a non-trivial way.
\end{abstract}




\section{INTRODUCTION}

Standard quantum theory is a remarkably successful theory. Indeed, there is at present not one shred of experimental evidence that its basic structure of Hilbert spaces, states, operators etc. is in any way wrong. However, in its normal presentation, the Copenhagen interpretation [1], it rests on certain assumptions that may be restrictive. Firstly, it relies on a division into classical and quantum domains. And secondly, it places great emphasis on the notion of measurement [2]. These assumptions are not of course a restriction in the regimes to which it is normally applied. Yet it invites the question, can we do better? Can we formulate standard quantum theory in such a way that it does not rely on these assumptions?

There are a number of reasons why one might want such a more general formulation. Recent experiments have started to probe the traditional border between what is normally called classical and quantum [3]. Furthermore, classical objects are after all built out of atoms, which are quantum mechanical in many of their properties. One can therefore ask how does classical behaviour emerge on large scales for objects made out of small quantum constituents? In addition, some current views of the early universe, and in particular in the area of research known as quantum cosmology, it is supposed that all force and matter fields are subject to the laws of quantum theory. There is no classical domain and there are certainly no measuring devices. How then can we understand, in a truly quantummechanical way, how measurements made in the present epoch are related to events in the distant past?

The decoherent histories approach to quantum theory is a reformulation of standard quantum theory for closed quantum systems (such as the entire universe), that removes the usual emphasis on the notion of measurement and of a classical domain. In this contribution I will briefly describe the formalism and the way it explains the emergence of classical physics

from an underlying quantum theory. I will then also describe a selection of applications of the decoherent histories approach in a variety of different circumstances. This will show what sort of light the approach has been able to shed on other fields. This review is by no means an exhaustive survey of the field, which has by now become quite extensive, and the choice of topics covered largely reflects my own interests in the subject. 


\section{INTERFERENCE, DECOHERENCE AND CLASSICALITY}

Before embarking on a full discussion of the decoherent histories formalism, let us first consider in a simple way the phenomenon of interference in the double slit experiment, its destruction through decoherence, and the relationship of this to emergent classicality.

Consider then the standard double slit experiment, in which electrons, say, impinge on a sheet with two slits and are then allowed to fall on a screen. The wave function is assumed to be a tightly peaked wave packet in the direction perpendicular to the screen, but spreads out in the direction parallel to it. In the region of the slits, we may assign a wave function

$$
\psi=\psi_{1}+\psi_{2}
$$

to the electrons, where $\psi_{1}$ represents a wave emerging from slit 1 , and $\psi_{2}$ represents a wave emerging from slit 2. The probability of hitting the screen at point $x$ is then

$$
\begin{aligned}
p(x) & =|\psi(x)|^{2} \\
& =\left|\psi_{1}\right|^{2}+\left|\psi_{2}\right|^{2}+\psi_{1} \psi_{2}^{*}+\psi_{1}^{*} \psi_{2}
\end{aligned}
$$

That is, the probability of hitting the screen at point $x$ is not the sum of the probabilities of the two separate paths taken by the electron, as it would be in the classical case. The probability sum rules are changed by the presence of quantum interference. Quantum effects are therefore characterized by a failure of usual classical probability sum rules. Or differently put, this corresponds to a failure of Boolean logic.

Note also the appearance of histories at this elementary level. The interference effect arises as a result of the fact that we are trying to talk about the properties of the electron at both the screen and the slits, that is, at two moments of time. This simple aspect is rarely mentioned in elementary textbooks, but turns out to be significant, as we shall see.

We see from this simple but important example that for a quantum system to become classical, interference terms must be destroyed. How does this come about? It is known in the double slit experiment that if we measure the position of the electron close to the slits, then the wave function will collapse into one of the states $\psi_{1}$ or $\psi_{2}$ and the interference terms will go away. We therefore expect that any physical mechanism that constitutes some kind of physical measurement of the electron will produce a similar effect. This is indeed

the case. Suppose, following the original calculation of Joos and Zeh we couple the electrons 
to an environment [4]. This could be, for example, a bath of photons. If the total wave function of the system (electron) together with its environment is denoted by $\left|\psi_{S \mathcal{E}}\right\rangle$, then the density operator of the system only is

$$
\rho=\operatorname{Tr}\left|\psi_{S \mathcal{E}}\right\rangle\left\langle\psi_{S \mathcal{E}}\right|
$$

where $\operatorname{Tr}$ denotes a trace over the environment. Such a density operator usually obeys a non-unitary master equation. It typically has the form (in one dimension),

$$
\frac{\partial \rho}{\partial t}=\frac{i \hbar}{2 M}\left(\frac{\partial^{2} \rho}{\partial x^{2}}-\frac{\partial^{2} \rho}{\partial y^{2}}\right)-D(x-y)^{2} \rho
$$

The important term is the last one on the right, which has the effect of causing the density operator $\rho(x, y)$ to become approximately diagonal in position very rapidly. The means that an initial state of the form

$$
\rho=|\psi\rangle\langle\psi|
$$

where

$$
|\psi\rangle=\left|\psi_{1}\right\rangle+\left|\psi_{2}\right\rangle
$$

becomes essentially indistinguishable from the classical mixture

$$
\rho=\left|\psi_{1}\right\rangle\left\langle\psi_{1}|+| \psi_{2}\right\rangle\left\langle\psi_{2}\right|
$$

This is the phenomenon of decoherence, in its simplest form.

Consider now the question of how a quantum system, characterized by its positions at a series of times, may become approximately classical. Quantum theory supplies probabilities, so suppose we use it to compute the probability $p\left(\alpha_{1}, t_{1}, \alpha_{2}, t_{2}, \cdots\right)$ that the a particle is in a spatial region $\alpha_{1}$ at time $t_{1}$, and then in a spatial region $\alpha_{2}$ at times $t_{2}$, etc. Then, "approximately classical" means at least two things. Firstly, it means that the probability is defined. As we have seen, interference can prevent this from being the case. Secondly, it means that the probability is strongly peaked when the regions $\alpha_{1}, \alpha_{2}, \alpha_{3} \cdots$ lie along a classical trajectory [5].

From this we see that histories provide an important mode of description for discussing emergent classicality. It is therefore perhaps not surprising that all of the above is most clearly formulated in terms of the decoherent histories approach. 


\section{THE DECOHERENT HISTORIES APPROACH}

The decoherent (or "consistent") histories approach was put forward by Griffiths in 1984 [6] whose work was substantially developed by Omnès [7, 8, 9]. It was also discovered and developed, in part independently, by Gell-Mann and Hartle [10, 11, 12]. See Ref.[13] for a very extensive bibliography on decoherent histories, and decoherence generally.

\section{A. The Formalism}

In the decoherent histories approach to quantum theory, a quantum-mechanical history is characterized by an initial (pure or mixed) state $\rho$, and by a time-ordered string of projection operators,

$$
C_{\underline{\alpha}}=P_{\alpha_{n}}\left(t_{n}\right) \cdots P_{\alpha_{1}}\left(t_{1}\right)
$$

Here, the projectors appearing in (8) are in the Heisenberg picture,

$$
P_{\alpha_{k}}\left(t_{k}\right)=e^{\frac{i}{\hbar} H\left(t_{k}-t_{0}\right)} P_{\alpha_{k}} e^{-\frac{i}{\hbar} H\left(t_{k}-t_{0}\right)}
$$

and $\underline{\alpha}$ denotes the string of alternatives $\alpha_{1}, \cdots \alpha_{n}$. The projection operators $P_{\alpha}$ characterize the different alternatives describing the histories at each moment of time. The projectors satisfy

$$
\sum_{\alpha} P_{\alpha}=1, \quad P_{\alpha} P_{\beta}=\delta_{\alpha \beta} P_{\alpha}
$$

More generally, it is also of interest to work with histories in which the so-called class

operators (8) are given by sums of string of projections (which are not necessarily then equal to strings of projections). Since the class operator (8) is not in fact a projection operator, it is reasonable to ask why this operator should be used to describe a history. This and many other related issues have been considered in detail by Isham and collaborators [14].

Probabilities are assigned to histories of a closed system via the formula,

$$
p\left(\alpha_{1}, \alpha_{2}, \cdots \alpha_{n}\right)=\operatorname{Tr}\left(C_{\underline{\alpha}} \rho C_{\underline{\alpha}}^{\dagger}\right)
$$

Interference between pairs of histories is measured by the decoherence functional

$$
D\left(\underline{\alpha}, \underline{\alpha}^{\prime}\right)=\operatorname{Tr}\left(C_{\underline{\alpha}} \rho C_{\underline{\alpha}^{\prime}}^{\dagger}\right)
$$


Probabilities can be assigned to histories if and only if all pairs of histories in the set obey the condition of consistency, which is that

$$
\operatorname{Re} D\left(\underline{\alpha}, \underline{\alpha}^{\prime}\right)=0
$$

for $\underline{\alpha} \neq \underline{\alpha}^{\prime}$. This condition is equivalent to the requirement that all probabilities satisfy the probability sum rules. These, loosely speaking, are that the probability of history $\underline{\alpha}$ or history $\underline{\beta}$ (for two disjoint histories), should be $p(\underline{\alpha})+p(\underline{\beta})$. Typically, realistic physical mechanisms which bring about the consistency condition (13) also cause the imaginary part of $D\left(\underline{\alpha}, \underline{\alpha}^{\prime}\right)$ to vanish for $\underline{\alpha} \neq \underline{\alpha}^{\prime}$ as well, so it is of interest to work with the stronger condition of decoherence, which is

$$
D\left(\underline{\alpha}, \underline{\alpha}^{\prime}\right)=0
$$

for $\underline{\alpha} \neq \underline{\alpha}^{\prime}$. The two conditions of consistency and decoherence have different consequences, and we discuss them below.

When the decoherence condition is satisfied, it is straightforward to see that

$$
p(\underline{\alpha})=\operatorname{Tr}\left(C_{\underline{\alpha}} \rho\right)
$$

since we have $\sum_{\underline{\alpha}} C_{\underline{\alpha}}=1$. Eq.(17) is clearly positive when the decoherence condition is satisfied, but not generally not otherwise. Goldstein and Page have turned this around and suggested that positivity of the expression (17) may be used to select physically viable histories [15]. This is clearly weaker than the usual decoherence condition. A variety of decoherence conditions related to those discussed above were also discussed in Ref.[16].

\section{B. Approximate Decoherence}

Typically, the decoherence condition Eq.(14) is satisfied only approximately, raising the question of what approximate decoherence actually means and how small the off-diagonal terms really need to be. In this context, it is worth noting that the decoherence functional satisfies the inequality,

$$
\left|D\left(\underline{\alpha}, \underline{\alpha}^{\prime}\right)\right|^{2} \leq D(\underline{\alpha}, \underline{\alpha}) D\left(\underline{\alpha}^{\prime}, \underline{\alpha}^{\prime}\right)
$$

as shown in Ref.[17]. A natural approximate decoherence condition is then to insist that the decoherence functional satisfies Eq.(17) but with a small number $\epsilon$ on the right-hand side. It may be shown that this also guarantees that most of the probability sum rules are satisfied to 
order $\epsilon^{1 / 2}$ [17]. On the more general issue of approximate decoherence, it has been suggested that an approximately decoherent set of histories may be turned into an exactly decoherent set by small distortions of the histories, for example, by small distortions of the operators projected onto at each moment of time [18]. A closely related suggestion, which has been worked out in some detail in particular cases, is that approximately decoherent histories are those whose predictions are well-approximated by a hidden variable theory, such as the deterministic quantum theory of 't Hooft [19]. See also Ref.[20] for further considerations of approximate decoherence.

\section{Path Integral Form}

It is also useful to note that for histories characterized by projections onto position at different time, the decoherence functional is very usefully expressed in terms of a path integral:

$$
D\left(\underline{\alpha}, \underline{\alpha}^{\prime}\right)=\int_{\underline{\alpha}} \mathcal{D} x \int_{\underline{\alpha}^{\prime}} \mathcal{D} y \exp \left(\frac{i}{\hbar} S[x(t)]-\frac{i}{\hbar} S[y(t)]\right) \rho_{0}\left(x_{0}, y_{0}\right)
$$

The path integral is over a pair of paths $x(t), y(t)$ which are folded into the initial state $\rho$ at $x_{0}, y_{0}$, wmeet at the final time (and the final point is summed over), and at intermediate times pass through the series of pairs of regions denoted by $\underline{\alpha}$ and $\underline{\alpha}^{\prime}$. This form is particularly useful for generalizations to situations which involve time in a non-trivial way.

\section{Consistency}

Consistency, Eq.(13), is an interesting condition, because, according to a theorem of Omnès, systems described by a set of consistent histories from a representation of classical logic [7]. That is, in a consistent set of histories each history corresponds to a proposition about the properties of a physical system and we can manipulate these propositions without contradiction using ordinary classical logic. In simple terms, we can talk about their properties. It is for this reason that the decoherent histories approach may be thought of as supplying a foundation for the application of ordinary reasoning to closed quantum systems. In the Copenhagen interpretation, it was asserted that one could only talk about measured quantities in an unambiguous way. In the decoherent histories approach, the idea of a a measured quantity is replaced by the weaker and more general idea of consistency. 
An important example is the case of retrodiction of the past from present data. Suppose we have a consistent set of histories. We would say that the alternative $\alpha_{n}$ (measured present data) implies the alternatives $\alpha_{n-1} \cdots \alpha_{1}$ (unmeasured past events) if

$$
p\left(\alpha_{1}, \cdots \alpha_{n-1} \mid \alpha_{n}\right) \equiv \frac{p\left(\alpha_{1}, \cdots \alpha_{n}\right)}{p\left(\alpha_{n}\right)}=1
$$

In this way, we can in quantum mechanics build a picture of the history of the universe, given the present data and the initial state, using only logic and the consistency of the histories. We can meaningfully talk about the past properties of the universe even though there was no measuring device there to record them. This is one reason why the histories approach is of interest in quantum cosmology.

\section{E. Decoherence and Records}

The stronger condition of decoherence is perhaps more interesting since it is related to the existence of records - some physical mechanism existing at a fixed moment of time which is correlated with the past history of the system. An example is a photographic plate showing a particle track. In particular, if the initial state is pure, there exist a set of records at the final time $t_{n}$ which are perfectly correlated with the alternatives $\alpha_{1} \cdots \alpha_{n}$ at times $t_{1} \cdots t_{n}$ [11]. This follows because, with a pure initial state $|\Psi\rangle$, the decoherence condition implies

that the states $C_{\underline{\alpha}}|\Psi\rangle$ are an orthogonal set. It is therefore possible to introduce a projection operator $R_{\underline{\beta}}$ (which is generally not unique) such that

$$
R_{\underline{\beta}} C_{\underline{\alpha}}|\Psi\rangle=\delta_{\underline{\alpha} \underline{\beta}} C_{\underline{\alpha}}|\Psi\rangle
$$

It follows that the extended histories characterized by the chain $R_{\underline{\beta}} C_{\underline{\alpha}}|\Psi\rangle$ are decoherent, and one can assign a probability to the histories $\underline{\alpha}$ and the records $\underline{\beta}$, given by

$$
p\left(\alpha_{1}, \alpha_{2}, \cdots \alpha_{n} ; \beta_{1}, \beta_{2} \cdots \beta_{n}\right)=\operatorname{Tr}\left(R_{\beta_{1} \beta_{2} \cdots \beta_{n}} C_{\underline{\alpha}} \rho C_{\underline{\alpha}}^{\dagger}\right)
$$

This probability is then zero unless $\alpha_{k}=\beta_{k}$ for all $k$, in which case it is equal to the original probability $p\left(\alpha_{1}, \cdots \alpha_{n}\right)$. Hence either the $\alpha$ 's or the $\beta$ 's can be completely summed out of Eq.(12) without changing the probability, so the probability for the histories can be entirely replaced by the probability for the records at a fixed moment of time at the end of the history:

$$
p(\underline{\alpha})=\operatorname{Tr}\left(R_{\underline{\alpha}} \rho\left(t_{n}\right)\right)=\operatorname{Tr}\left(C_{\underline{\alpha}} \rho C_{\underline{\alpha}}^{\dagger}\right)
$$


Conversely, the existence of records $\beta_{1}, \cdots \beta_{n}$ at some final time perfectly correlated with earlier alternatives $\alpha_{1}, \cdots \alpha_{n}$ at $t_{1}, \cdots t_{n}$ implies decoherence of the histories. This may be seen from the relation

$$
D\left(\underline{\alpha}, \underline{\alpha}^{\prime}\right)=\sum_{\beta_{1} \cdots \beta_{n}} \operatorname{Tr}\left(R_{\beta_{1} \cdots \beta_{n}} C_{\underline{\alpha}} \rho C_{\underline{\alpha}^{\prime}}^{\dagger}\right)
$$

Since each $\beta_{k}$ is perfectly correlated with a unique alternative $\alpha_{k}$ at time $t_{k}$, the summand

on the right-hand side is zero unless $\alpha_{k}=\alpha_{k}^{\prime}$ (although note that, as we shall see later, a perfect correlation of this type is generally possible only for a pure initial state).

There is, therefore, a very general connection between decoherence and the existence of records. From this point of view, the decoherent histories approach is very much concerned with reconstructing possible past histories of the universe from records at the present time, and then using these reconstructed pasts to understand the correlations amongst the present records [21].

Some explicit models where the records may be explicitly identified have been worked out. Ref.[22] showed how the environment stores records of the particle's history in the quantum Brownian motion model (in which the environment is a set of harmonic oscillators). This was repeated for the case of decoherence by a series of scattering processes in Ref.[23].

\section{F. The Non-Uniqueness of Retrodiction}

The above scheme contains an important subtlety that we now need to discuss. This is that the retrodicted past is not in fact unique: there are often many sets of consistent histories associated with the same initial state and final measurement, and what's more, can give conditional probability 1 for different complementary observables at an intermediate time. To see how this happens, consider the following example (due to Omnès.) Suppose we have a radioactive atom sitting at the origin which decays at $t=0$, and therefore emits a particle in an outgoing spherical wave state, and then hits a detector at time $t=t_{2}$. It is then reasonable to ask if we can say anything about the system at an intermediate time $t_{1}$.

We may analyze this using the decoherent histories approach. The appropriate decoherence functional is,

$$
D\left(\alpha_{1}, \alpha_{2} \mid \alpha_{1}^{\prime}, \alpha_{2}\right)=\operatorname{Tr}\left(P_{\alpha_{2}}\left(t_{2}\right) P_{\alpha_{1}}\left(t_{1}\right) \rho P_{\alpha_{1}^{\prime}}\left(t_{1}\right)\right)
$$

Here, $P_{\alpha_{2}}$ is a projector onto the position of the detector, $\rho=|\psi\rangle\langle\psi|$ is the spherical wave 
state, and $P_{\alpha_{1}}$ is a projector onto possible properties we may measure at the intermediate time $t_{1}$. We consider two cases.

First, suppose that we project at time $t_{1}$ onto a spatial region lying between the detector and the origin. Then, one finds quite easily that the histories are approximately decoherent, and secondly, that the conditional probability $p\left(\alpha_{1} \mid \alpha_{2}\right)$ of finding the particle at $\alpha_{1}$, given that it was at $\alpha_{2}$ is approximately equal to 1 . That is, if the detector clicks, then we may logically deduce that the particle followed a trajectory in the past, along the direct line from the origin to the detector.

Second, suppose we project instead onto a completely different intermediate state. The initial state is an outgoing spherical wave, and we there expect that there will be consistent histories reflecting this fact. Suppose we therefore project onto this possibility, using the projector,

$$
P_{\alpha_{1}}\left(t_{1}\right)=\left|\psi\left(t_{1}\right)\right\rangle\left\langle\psi\left(t_{1}\right)\right|
$$

(together with its negation). Then we again get consistency, and that $p\left(\alpha_{1} \mid \alpha_{2}\right)=1$, from which we would be inclined to say that the particle was in a spherical wave state at time $t_{1}$. A third possibility would be to project onto the momentum at $t_{1}$, which would lead to yet another set of consistent histories, in which the value of momentum is predicted with near certainty.

This illustrates that there are different sets of consistent histories for the same physical situation, depending on which variables one would like to talk about. Moreover, these different choices for $P_{\alpha_{1}}\left(t_{1}\right)$ do not commute, and this means that if we attempted to combine them in a single set, the consistency condition would no longer be satisfied, and we would not be allowed to make any logical deductions. This feature of the formalism is essentially quantum-mechanical complementarity, although it appears in a form which is for some quite disconcerting. It raises the question as to whether one would be able to assign definite values to non-commuting observables. This, however, is excluded by a rule proposed by Griffiths [6], which states that all logical deductions about the system must be made from the framework of a single consistent set.

The non-uniqueness of the retrodicted past then raises some questions as to the value of the whole formalism, and what practical use it is. It means that quantum theory, in the decoherent histories version of it, does not uniquely tell us what "actually happened", although to be sure, this feature of quantum theory is certainly known already in various 
ways. See Refs. $[9,18,24,25]$ for disucussions of these issues.

The value of the formalism becomes clear when we ask what do we actually do with the retrodicted past. The answer is that we use it to make other predictions about the present. That is, we start from some present data (measurements or cosmological observations), and, using consistency or decoherence, we retrodict the past. We then use the retrodicted past to make more predictions about the present. The role of the histories, therefore, is that they are an intermediate tool which helps us to identify correlations between data sets at a fixed moment of time. For example, when we look at a photograph of a particle track, we see a series of dots, existing at a fixed moment of time, which appear to be correlated. The explanation of their correlation is to be found in appealing to the past history of the system that produced the dots. Differently put, by looking, for example, at just three dots, we can use decoherence and retrodiction to deduce that a particle passed through on an approximately classical history. We then use the history to correctly deduce the location of the remaining dots on the photographic plate. In brief, therefore, histories are a useful tool to help us understand present records [21].

\section{QUANTUM BROWNIAN MOTION MODEL}

We now briefly consider a particular model, namely the quantum Brownian motion model. This model has been extensively studied in the literature so only the briefest of accounts will be given here [17]. The model consists of a particle of mass $M$ in a potential $V(x)$ linearly coupled to an environment consisting of a large bath of harmonic oscillators in a thermal state at temperature $T$ [26]. We consider histories of position samplings of the distinguished system. The samplings are continuous in time and Gaussian sampling functions are used (corresponding to approximate projection operators). The decoherence functional for the model is most conveniently given in path-integral form:

$$
\begin{aligned}
D[\bar{x}(t), \bar{y}(t)] & =\int \mathcal{D} x \mathcal{D} y \delta\left(x_{f}-y_{f}\right) \rho\left(x_{0}, y_{0}\right) \\
& \times \exp \left(\frac{i}{\hbar} S[x(t)]-\frac{i}{\hbar} S[y(t)]+\frac{i}{\hbar} W[x, y]\right) \\
& \times \exp \left(-\int d t \frac{(x(t)-\bar{x}(t))^{2}}{2 \sigma^{2}}-\int d t \frac{(y(t)-\bar{y}(t))^{2}}{2 \sigma^{2}}\right)
\end{aligned}
$$

Here, $S$ is the action for a particle in a potential $V(x), \bar{x}(t), \bar{y}(t)$ are the sampled positions and $x_{f}$ and $x_{0}$ denote the final and initial values respectively. The effects of the environment 
are summarized entirely by the Feynman-Vernon influence functional phase, $W[x, y]$, given by,

$$
\begin{aligned}
W[x(t), y(t)] & =-\int_{0}^{t} d s \int_{0}^{s} d s^{\prime}[x(s)-y(s)] \eta\left(s-s^{\prime}\right)\left[x\left(s^{\prime}\right)+y\left(s^{\prime}\right)\right] \\
& +i \int_{0}^{t} d s \int_{0}^{s} d s^{\prime}[x(s)-y(s)] \nu\left(s-s^{\prime}\right)\left[x\left(s^{\prime}\right)-y\left(s^{\prime}\right)\right]
\end{aligned}
$$

The explicit forms of the non-local kernels $\eta$ and $\nu$ may be found, for example, in Ref.[27]. Here it is assumed, as is typical in these models, that the initial density matrix of the total system is simply a product of the initial system and environment density matrices, and the initial environment density matrix is a thermal state at temperature $T$. Considerable simplifications occur in a purely ohmic environment in the Fokker-Planck limit (a particular form of the high temperature limit), in which one has

$$
\begin{aligned}
& \eta\left(s-s^{\prime}\right)=M \gamma \delta^{\prime}\left(s-s^{\prime}\right) \\
& \nu\left(s-s^{\prime}\right)=\frac{2 M \gamma k T}{\hbar} \delta\left(s-s^{\prime}\right)
\end{aligned}
$$

where $\gamma$ is the dissipation. For convenience we will work in this limit. One can see almost immediately that the imaginary part of $W$, together with the Gaussian samplings in Eq.(25), will have the effect of suppressing widely differing paths $\bar{x}(t), \bar{y}(t)$. Indeed, the suppression factor will be of order

$$
\exp \left(-\frac{2 M \gamma k T \sigma^{2}}{\hbar^{2}}\right)
$$

In cgs units $\hbar \sim 10^{-27}$ and $k \sim 10^{-16}$, so $k T / \hbar^{2} \sim 10^{40}$ if $T$ is room temperature. Values of order 1 for $M, \gamma$ and $\sigma$ therefore lead to an astoundingly small suppression factor. Decoherence through interaction with a thermal environment is thus a very effective process indeed.

More precisely, one can approximately evaluate the functional integral Eq.(25). Let $X=(x+y) / 2, \xi=x-y$, and use the smallness of the suppression factor to expand about $\xi=0$. Then the $\xi$ functional integral may be carried out with the result,

$$
\begin{aligned}
D[\bar{x}(t), \bar{y}(t)] & =\int \mathcal{D} X W\left(M \dot{X}_{0}, X_{0}\right) \exp \left(-\int d t \frac{\left(X-\frac{\bar{x}+\bar{y}}{2}\right)^{2}}{\sigma^{2}}\right) \\
& \times \exp \left(-\int d t \frac{F[X]^{2}}{2(\Delta F)^{2}}-i \hbar \int d t \frac{(\bar{x}-\bar{y}) F[X]}{4 \sigma^{2}(\Delta F)^{2}}\right) \\
& \times \exp \left(-\int d t \frac{(\bar{x}-\bar{y})^{2}}{2 \ell^{2}}\right)
\end{aligned}
$$


where

$$
F[X]=M \ddot{X}+M \gamma \dot{X}+V^{\prime}(X)
$$

are the classical field equations with dissipation, and

$$
\begin{aligned}
(\Delta F)^{2} & =\frac{\hbar^{2}}{\sigma^{2}}+4 M \gamma k T \\
\ell^{2} & =2 \sigma^{2}+\frac{\hbar^{2}}{4 M \gamma k T}
\end{aligned}
$$

$W\left(M \dot{X}_{0}, X_{0}\right)$ is the Wigner transform of the initial density operator.

The decoherence width Eq.(33) does not, in fact, immediately indicate the expected suppression of interference, because the temperature-dependent term will typically be utterly negligible compared to the $\sigma^{2}$ term. The point, however, is that more precise notions of decoherence need to be employed. One should check some of the probability sum rules, or use the approximate decoherence condition discussed in Ref.[17], in which the sizes of the off and on-diagonal terms are compared. This has not been carried out for the general expression (30), and in fact seems to be rather hard. Satisfaction of the approximate decoherence condition was checked for some special cases in Ref.[17]. Still, one expects the standard to which decoherence is attained to be of the order of the suppression factor (29), i.e., very good indeed.

Now consider the diagonal elements of the decoherence function, representing the probabilities for histories.

$$
\begin{aligned}
p[\bar{x}(t)] & =\int \mathcal{D} X W\left(M \dot{X}_{0}, X_{0}\right) \\
& \times \exp \left(-\int d t \frac{(X-\bar{x})^{2}}{\sigma^{2}}-\int d t \frac{F[X]^{2}}{2(\Delta F)^{2}}\right)
\end{aligned}
$$

The distribution is peaked about configurations $\bar{x}(t)$ satisfying the classical field equations with dissipation; thus approximate classical predictability is exhibited. The width of the peak is given by (32). Loosely speaking, a given classical history occurs with a weight given by the Wigner function of its initial data. This cannot be strictly correct, because the Wigner function is not positive in general, although it is if coarse-grained over an $\hbar$-sized region of phase space [28].

The width (32) has clearly identifiable contributions from quantum and thermal fluctuations. The thermal fluctuations dominate the quantum ones when $8 M \gamma k T \sigma^{2}>\hbar^{2}$, which, from (29), is precisely the condition required for decoherence, as previously noted. 
Environmentally-induced fluctuations are therefore inescapable if one is to have decoherence. This means that there is a tension between the demands of decoherence and classical predictability, both of which are necessary (although generally not sufficient) for the emergence of a quasiclassical domain [11]. This tension is due to the fact that the degree of decoherence (29) improves with increasing environment temperature, but predictability deteriorates, because the fluctuations (32) grow. However, the smallness of Boltzmann's constant ensures that the fluctuations (32) will be small compared to $F[X]$ for a wide range of temperatures if $M$ is sufficiently large. Moreover, the efficiency of decoherence as evidenced through (29) is largely due to the smallness of $\hbar$, and will hold for a wide range of temperatures. So although there is some tension, there is a broad compromise regime in which decoherence and classical predictability can each hold extremely well.

One might wonder what sort of restrictions the uncertainty principle places on the degree to which probabilities for histories may be peaked about a particular history. For clearly it is not possible to be perfectly peaked because that would mean definite values for positions at different times, which are non-commuting operators. It turns out that the Shannon information,

$$
I=\sum_{\underline{\alpha}} p(\underline{\alpha}) \ln p(\underline{\alpha})
$$

provides a useful measure of the degree of peaking, and the uncertainty principle appears as a lower bound on $I$, as shown in Ref.[29].

\section{QUANTUM CLASSICAL COUPLINGS}

An interesting further development of the previous section concerns the construction of consistent theories describing the interaction of classical and quantum systems. This section primarily follows Ref.[30]. (See also Ref.[31].) This is a question of interest in a variety of different areas, in particular, in quantum field theory in curved spacetime, where one is interesting in assessing the effect a quantum field has on a classical gravitational field. To be more precise, let us consider the simpler case of a massive particle of mass $M$, position $X$ and velocity $\dot{X}$. Suppose this particle, which we take to be well-described by classical mechanics, comes into interaction with a particle of mass $m$, which is sufficiently light that it is predominantly quantum-mechanical and described by a state vector $|\psi\rangle$. What happens to the classical particle under these conditions? 
With a simple linear coupling between these systems, the classical particle obeys the equation of motion,

$$
M \ddot{X}+V^{\prime}(X)+\lambda x=0
$$

and the state of the quantum particle obeys the equation

$$
i \hbar \frac{d|\psi\rangle}{d t}=H_{X}|\psi\rangle
$$

where $H_{X}$ denotes the quantum particle's Hamiltonian in the presence of a classical external field $X$. The problem with this system, however, is how to interpret the quantity $x$ in Eq.(36), which clearly should be an operator because it describe a quantum system. The simplest suggested resolution to this is to insert a quantum-mechanical expectation value, and to use instead of (36) the equation

$$
M \ddot{X}+V^{\prime}(X)+\lambda\langle\psi|x| \psi\rangle=0
$$

However, one would expect this prescription to yield reasonable results only in a limited set of circumstances. Indeed, it gives physically unreasonable results in the interesting case when the quantum particle is in a superposition of localized position states. Clearly in that case the correct physical answer is that the classical particle should "see" one or other of the localized superposition states, and not some kind of averaged position, which is what (38) indicates.

An elementary extension of the results of the previous section may be used to construct a more sensible coupled classical-quantum system. One may start from the assumption that there are no truly classical systems, only quantum systems that are approximately classical under certain circumstances, as discussed in the previous section. Consider therefore, a large particle of mass $M$ and position $X$ coupled to an environment, to make it approximately classical. Suppose also that the massive particle is again coupled to the light particle, as in Eq.(36). We now look for decoherent histories of the massive particle.

It is easily shown that there are decoherent histories of positions of the massive particle, whose probabilities are strongly peaked about the equation of motion,

$$
M \ddot{X}+M \gamma \dot{X}+V^{\prime}(X)+\lambda \bar{x}=0
$$

These are the classical equations of motion with dissipation, as expected (and also with fluctuations, encoded in the width of the peak). The interesting extra ingredient, however, 
is the quantity $\bar{x}$ which is now not an operator, but a stochastic c-number, for which the decoherent histories approach supplies a probability distribution function $p[\bar{x}(t)]$. This is a physically sensible result: the classical particle responds in a stochastic way to the quantum system. Furthermore, this construction gives the expected sensible results for superposition states of the quantum particle.

\section{DECOHERENT HISTORIES AND QUANTUM STATE DIFFUSION}

For the particular, yet commonly realized situation in which there is a natural split into system and environment, it turns out that the decoherent histories approach is closely related to the quantum state diffusion approach to open quantum systems. We begin be briefly explaining what this is.

Very many open quantum systems are accurately described by the Lindblad master equation for the reduced density operator $\rho$ [32]. This is

$$
\frac{d \rho}{d t}=-\frac{i}{\hbar}[H, \rho]-\frac{1}{2} \sum_{j=1}^{n}\left(\left\{L_{j}^{\dagger} L_{j}, \rho\right\}-2 L_{j} \rho L_{j}^{\dagger}\right)
$$

Here, $H$ is the Hamiltonian of the open system in the absence of the environment (sometimes modified by terms depending on the $L_{j}$ ) and the $n$ operators $L_{j}$ model the effects of the environment. The Lindblad form is the most general possible evolution equation preserving positivity, hermiticity and trace, subject only to the physically useful assumption that the evolution is Markovian. For example, in the quantum Brownian motion model, the master equation has a single non-hermitian $L$ which is a linear combination of position and momentum operators, and the Markovian approximation is valid for reasonably high temperatures.

Whilst the master equation is the correct quantum-mechanical description for many open systems, its solutions do not easily yield the expected physical picture that may be directly compared with experiments. Take again the case of quantum Brownian motion. There, coarse observations of the system yield trajectories in phase space following approximately classical paths with dissipation and fluctuations. Yet an initial localized wavepacket will spread indefinitely as a result of quantum and thermal fluctuations under evolution according to Eq.(40). Differently put, the density operator really corresponds to an ensemble of trajectories, yet an experiment measures an individual trajectory of the system. 
The quantum state diffusion picture, introduced by Gisin and Percival [33], aimed to expose the individual trajectories contained in the density operator equation. In this picture, the density operator $\rho$ satisfying (40) is regarded as a mean over a distribution of pure state density operators,

$$
\rho=M|\psi\rangle\langle\psi|
$$

where $M$ denotes the mean (defined below), with the pure states evolving according to the non-linear stochastic Langevin-Ito equation,

$$
\begin{aligned}
|d \psi\rangle & =-\frac{i}{\hbar} H|\psi\rangle d t+\frac{1}{2} \sum_{j}\left(2\left\langle L_{j}^{\dagger}\right\rangle L_{j}-L_{j}^{\dagger} L_{j}-\left\langle L_{j}^{\dagger}\right\rangle\left\langle L_{j}\right\rangle\right)|\psi\rangle d t \\
& +\sum_{j}\left(L_{j}-\left\langle L_{j}\right\rangle\right)|\psi\rangle d \xi_{j}(t)
\end{aligned}
$$

for the normalized state vector $|\psi\rangle$. Here, the $d \xi_{j}$ are independent complex differential random variables representing a complex Wiener process. Their linear and quadratic means are,

$$
M\left[d \xi_{j} d \xi_{k}^{*}\right]=\delta_{j k} d t, \quad M\left[d \xi_{j} d \xi_{k}\right]=0, \quad M\left[d \xi_{j}\right]=0
$$

This equation is very similar to the explicit modified versions of quantum theory, such as that due to Ghirardi et al.[34], but here the motivation is different, in that the stochastic equation is not proposed as a fundamental equation.

An interesting feature of Eq.(42) is that its solutions tend to undergo some kind of localization in time, quite the opposite of the master equation. For example, it may be shown that in the quantum Brownian motion model, all initial states become localized around a wave packet tightly peaked in phase space after a very short time, and thereafter remain localized and follow the classical equations of motion [33, 35]. Hence the quantum state diffusion approach naturally provides the intuitive appealing and experimentally correct picture of an individual trajectory. Furthermore, one can also calculate a probability for each trajectory.

It is therefore easily seen that both of the intuitive picture and physically predictions of quantum state diffusion are in fact essentially the same as those provided by the decoherent histories approach. For the histories approach also naturally provides the picture of an individual trajectory, and a probability for those trajectories. This is argued in much greater detail in Ref.[36]. Differently put, the solutions to quantum state diffusion in some sense represent a single history from a decoherent set. Also, QSD was put forward as a 
phenomenological picture of open quantum systems. This connection with the decoherent histories approach may be thought of as a more fundamental justification of QSD phenomenology.

More general versions of this connection, involving other types of stochastic unravelings have been discovered by Brun [37].

\section{HYDRODYNAMIC EQUATIONS}

Most models of decoherence, such as the quantum Brownian motion model of Section 4, rely on an obvious separation of the whole closed system in a distinguished subsystem, and the rest (the environment). This is a reasonable assumption for a wide variety of physically interesting situations. The decoherent histories approach, however, does not rely on such a separation, and this is important, because there are situations or regimes where such a split does not necessarily exist. This then raises the question, in a large and possibly complex quantum system, with no obvious system-environment split, what are the variables that naturally become classical and what sorts of classical equations of motion emerge from the underlying quantum theory? Differently put, what is the most general possible derivation of emergent classicality?

Gell-Mann and Hartle have argued that one particular set of variables that are strong candidates for the "habitually decohering" variables are the integrals over small volumes of locally conserved densities [11]. These variables are distinguished by the existence of conservation laws for total energy, momentum, charge, particle number, etc. Associated with such conservation laws are local conservation laws of the form

$$
\frac{\partial \rho}{\partial t}+\nabla \cdot \mathbf{j}=0
$$

The candidate quasiclassical variables are then

$$
Q_{V}=\int_{V} d^{3} x \rho(\mathbf{x})
$$

If the volume $V$ over which the local densities are smeared is infinite, $Q_{V}$ will be an exactly conserved quantity. In quantum mechanics it will commute with the Hamiltonian, and, as is easily seen, histories of $Q_{V}$ 's will then decohere exactly [38]. If the volume is finite but large compared to the microscopic scale, $Q_{V}$ will be slowly varying compared to all 
other dynamical variables. This is because the local conservation law (44) permits $Q_{V}$ to change only by redistribution, which is limited by the rate at which the locally conserved quantity can flow out of the volume. Because these quantities are slowly varying, histories of them should therefore approximately decohere. Furthermore, the fact that the $Q_{V}$ 's are slowly varying may also be used, at least classically, to derive an approximately closed set of equations involving only those quantities singled out by the conservation laws. These equations are, for example, the Navier-Stokes equations, and the derivation of them is a standard (although generally non-trivial) exercise in non-equilibrium statistical mechanics [39].

One of the current goals of the decoherent histories approach is to carry this programme through in detail. A more detailed sketch of how this works was put forward in Refs. [40, 41] and a variety of models and related aspects are described in Ref.[42].

\section{SPACETIME COARSE GRAININGS}

Another class of questions to which the decoherent histories approach adapts very well are those that involve time in a non-trivial way. In particular, the arrival time and tunneling time problems have been the subject of considerable recent interest, that interesting stemming from the fact that quantum mechanics does not obviously supply a unique and physically reasonable prescription for addressing those problems. The decoherent histories approach offers yet another way to analyzing these questions.

To focus ideas, consider the question, what is the probability that a particle enters the spatial region $\Delta$ at any time, during the time interval $[0, \tau]$ ? This has been address by Yamada and Takagi [43], Hartle [44] and Micanek and Hartle [45], most of these concentrating on the case where $\Delta$ is the region $x<0$. The decoherence functional can be constructed using the path integral form Eq.(17) as a starting point. The fact that the question is nontrivial in time means that there is interference, and the decoherence or consistency conditions are satisfied only for very special choices of initial state, and the resultant probabilities are rather trivial. For example, in the case where $\Delta$ is $x<0$, the initial state must be antisymmetric about $x=0$, and the probability of entering the region is zero. In particular, this analysis does not admit the interesting case of a wave packet starting in $x>0$ and approaching the origin. Moreover, it does not even give results that have a sensible classical 
limit.

One can develop this approach further, and include an environment to produce decoherence, along the lines of the quantum Brownian motion model of Section 4 [46]. This then allows the assignment of probabilities for a wide variety of initial states, with a sensible and expected classical limit. However, they are essentially the probabilities one might anticipate on the basis of a classical Fokker-Planck equation for classical Brownian motion, and moreover, the probabilities seem to depend on the features of the environment.

At the present state of play, therefore, the results of the decoherent histories analysis are not very striking. There is, however, space for much more work to be done in this area. Furthermore, they appear to be compatible with other approaches to the arrival time problem, which involves, for example, time operators or detector models. The fact that most initial states do not satisfy the consistency condition (in the case of no environment) appears to be related to the fact that paths in the path integral move in and out of the region $\Delta$ many times, which in turn appears to be related to the fact that the time operators proposed in other approaches are not self-adjoint. Similarly, the dependence of the result on the details of the environment seems to be related to the fact that, in detector model approaches, the result depends on the form of the detector. See Ref.[47] for a more detailed review of these issues.

Finally, on the subject of temporal issues, it is worth noting that the so-called temporal Bell inequalities [48] may play in interesting role in the decoherent histories approach. The temporal Bell inequalities are a set of inequalities on certain probabilities or correlation functions which are derived by assuming that there exists a valid probability distribution for certain variables at a series of times. From the point of view of the decoherent histories approach, this assumption is only true when the histories are decoherent. The temporal Bell inequalities may therefore provide an interesting way of characterizing decoherence of histories, or the lack of decoherence, although this possibility does not seem to have received much attention to date.

\section{QUANTUM THEORY WITHOUT TIME}

An important extension of the ideas outlined in the previous section is the further extension of the decoherent histories approach to situations which do no involve specifying 
time in any way whatsoever. For example, in classical mechanics whether a particle follows a particular classical path. Or we can ask whether a particle's path enters a given region of space at stage along the entire trajectory. Such questions are interesting and relevant for quantum cosmology. There, the wave function of the system obeys not a Schrödinger equation, but the Wheeler-DeWitt equation, which has the form,

$$
H \Psi=0
$$

That is, the state function of the system is a zero energy eigenstate. The form of this equation arises as a result of reparametrization invariance, or more generally, the four-dimensional diffeomorphism invariance of general relativity. The problems of interpreting the solutions to this equation are similar to the problems of interpreting the Klein-Gordon equation as a first-quantized wave equation, and have attracted a considerable amount of interest over the years. Like the arrival time problem, there are a variety of different approaches to it. The decoherent histories approach is well-adapted to this problem. This is partly because, as we shall see, the natural reparametrization-invariant notion is an entire classical trajectory, and the histories approach handles this notion straightforwardly. We will briefly review the decoherent histories analysis of the question. The detailed analysis of the Klein-Gordon equation has been carried out in Ref.[49] and more general timeless models have been studied in Ref.[50]. We follow the latter quite closely.

To be precise, suppose we have an $n$ dimensional configuration space with coordinates $\mathbf{x}=\left(x_{1}, x_{2}, \cdots x_{n}\right)$, and suppose the wave function $\psi(\mathbf{x})$ of the system is in an eigenstate of the Hamiltonian, as in Eq.(46). What is the probability of finding the system in a region $\Delta$ of configuration space without reference to time? The classical case contains almost all the key features of the problem and we concentrate on this.

We will consider a classical system described by a $2 n$-dimensional phase space, with coordinates and momenta $(\mathbf{x}, \mathbf{p})=\left(x_{k}, p_{k}\right)$, and Hamiltonian

$$
H=\frac{\mathbf{p}^{2}}{2 M}+V(\mathbf{x})
$$

We assume that there is a classical phase space distribution function $w(\mathbf{p}, \mathbf{x})$, which is normalized according to

$$
\int d^{n} p d^{n} x w(\mathbf{p}, \mathbf{x})=1
$$


and obeys the evolution equation

$$
\frac{\partial w}{\partial t}=\sum_{k}\left(-\frac{p_{k}}{M} \frac{\partial w}{\partial x_{k}}+\frac{\partial V}{\partial x_{k}} \frac{\partial w}{\partial p_{k}}\right)=\{H, w\}
$$

where $\{$,$\} denotes the Poisson bracket. The interesting case is that in which w$ is the classical analogue of an energy eigenstate, in which case $\partial w / \partial t=0$, so the evolution equation is simply

$$
\{H, w\}=0
$$

It follows that

$$
w\left(\mathbf{p}^{c l}(t), \mathbf{x}^{c l}(t)\right)=w(\mathbf{p}(0), \mathbf{x}(0))
$$

where $\mathbf{p}^{c l}(t), \mathbf{x}^{c l}(t)$ are the classical solutions with initial data $\mathbf{p}(0), \mathbf{x}(0)$, so $w$ is constant along the classical orbits.

Given a set of classical solutions $\left(\mathbf{p}^{c l}(t), \mathbf{x}^{c l}(t)\right)$, and a phase space distribution function $w$, we are interested in the probability that a classical solution will pass through a region $\Delta$ of configuration space. To see whether the classical trajectory $\mathbf{x}^{c l}(t)$ intersects this region, consider the phase space function

$$
A\left(\mathbf{x}, \mathbf{p}_{0}, \mathbf{x}_{0}\right)=\int_{-\infty}^{\infty} d t \delta^{(n)}\left(\mathbf{x}-\mathbf{x}^{c l}(t)\right)
$$

(In the case of periodic classical orbits, the range of $t$ is taken to be equal to the period). This function is positive for points $\mathbf{x}$ on the classical trajectory labeled by $\mathbf{p}_{0}, \mathbf{x}_{0}$ and zero otherwise. It also has the property that

$$
\{H, A\}=0
$$

so is a reparametrization-invariant observable. This is the mathematical expression of the statement above that an entire classical trajectory is a reparametrization-invariant notion. Intersection of the classical trajectory with the region $\Delta$ means,

$$
\int_{\Delta} d^{n} x A\left(\mathbf{x}, \mathbf{p}_{0}, \mathbf{x}_{0}\right)>0
$$

The quantity on the left is essentially the amount of parameter time the trajectory spends in the region $\Delta$, so we are simply requiring it to be positive. We may now write down the probability for a classical trajectory entering the region $\Delta$. It is,

$$
p_{\Delta}=\int d^{n} p_{0} d^{n} x_{0} w\left(\mathbf{p}_{0}, \mathbf{x}_{0}\right) \theta\left(\int_{\Delta} d^{n} x A-\epsilon\right)
$$


In this construction, $\epsilon$ is a small positive number that is eventually sent to zero, and is included to avoid possible ambiguities in the $\theta$-function at zero argument. The $\theta$-function ensures that the phase space integral is over all initial data whose corresponding classical trajectories spend a time greater than $\epsilon$ in the region $\Delta$. Eq.(55) is the desired formula for the probability of entering the region $\Delta$ without regard to time. All elements of it are reparametrization invariant.

At the meeting, a possible difficulty with Eq.(54) was pointed out by T.Brun. This is that for chaotic systems, the trajectories visit every region in phase space, and it therefore appears that every trajectory will intersect the region $\Delta$. At present it therefore looks like the analysis presented here is valid only for integrable systems. A more general version of this analysis avoiding this difficulty is currently being sought, and will be described elsewhere. A possible solution is that asking whether the system intersects a given region of configuration space is not in fact a useful observable for chaotic systems, although there is no reason why there should not exist other types of observables that are useful.

Turning now to the quantum theory, a decoherence functional of the general form (12) may be constructed. Special attention is required for the inner product (since the solutions to (46) are typically not normalizable in the usual sense), and also for the class operators $C_{\underline{\alpha}}$, which must be reparametrization invariant and describe paths which pass through the region $\Delta$. Also, an environment is typically required in order to obtain decoherence. These non-trivial aspects were discussed at length in Ref.[50]. The final result, in essence, is that in the semiclassical limit, one obtains a formula of the form (55) in which the probability function $w$ is replaced by the Wigner function of the quantum state. Furthermore, this

formula may be rewritten in such a way that it coincides with earlier heuristic analyses of the Wheeler-DeWitt equation (such as the so-called WKB interpretation).

In summary, the decoherent histories approach successfully adapts to genuinely timeless systems, such as the Wheeler-DeWitt equation, although there is much more work to be done on this question.

\section{SUMMARY}

I have described the decoherent histories approach, its properties and some recent applications and developments. These may be summarized as follows: 
- The decoherent histories approach is essentially the Copenhagen approach to quantum mechanics, but relies on a smaller set of assumptions. In essence, it formalizes intuition.

- The approach gives a very comprehensive account of emergent classicality in a variety of situations, plus fluctuations about it, and couplings to quantum systems.

- It naturally accommodates situations where there is no system-environment split.

- It readily generalizes to situations which are non-trivial in time, or which do not involve time at all, as we expect to be the case in quantum gravity.

\section{ACKNOWLEDGEMENTS}

I am very grateful to Thomas Elze to inviting me to take part in this most stimulating meeting. I would also like to thank Todd Brun for useful comments on my talk at the meeting.

[1] A useful source of literature on the Copenhagen interpretation is, J. A. Wheeler and W. Zurek (eds.), Quantum Theory and Measurement (Princeton University Press, Princeton, NJ, 1983).

[2] J. Bell, Physics World 3, 33 (1990).

[3] A. J. Leggett, Suppl.Prog.Theor.Phys. 69, 80 (1980).

[4] E.Joos and H.D.Zeh, Z.Phys. B59, 223 (1985).

[5] J.B.Hartle, in, Proceedings of the Cornelius Lanczos International Centenary Confererence, edited by J.D.Brown, M.T.Chu, D.C.Ellison and R.J.Plemmons (SIAM, Philadelphia, 1994), also available as e-print gr-qc/9404017 (1994).

[6] R. B. Griffiths, J.Stat.Phys. 36, 219 (1984).

[7] R. Omnès, J.Stat.Phys. 53, 893 (1988).

[8] R. Omnès, J.Stat.Phys. 53, 933 (1988); 53, 957 (1988); 57, 357 (1989); Phys.Lett. A138, 157 (1989); Ann.Phys. 201, 354 (1990); Rev.Mod.Phys. 64, 339 (1992).

[9] R. Omnès, Phys.Lett. A187, 26 (1994); J.Stat.Phys. 62, 841 (1991).

[10] M. Gell-Mann and J. B. Hartle, in Complexity, Entropy and the Physics of Information, SFI Studies in the Sciences of Complexity, Vol. VIII, W. Zurek (ed.) (Addison Wesley, Reading, 1990); and in Proceedings of the Third International Symposium on the Foundations of Quan- 
tum Mechanics in the Light of New Technology, S. Kobayashi, H. Ezawa, Y. Murayama and S. Nomura (eds.) (Physical Society of Japan, Tokyo, 1990).

[11] M. Gell-Mann and J. B. Hartle, Phys.Rev. D47, 3345 (1993).

[12] J.B.Hartle, in Proceedings of the 1992 Les Houches Summer School, Gravitation et Quantifications, edited by B.Julia and J.Zinn-Justin (Elsevier Science B.V., 1995).

[13] T.Brun, Decoherence and Consistent Histories Reference List, www.sns.ias.edu/ tbrun.

[14] C. Isham, J.Math.Phys. 23, 2157 (1994); C. Isham and N. Linden, J.Math.Phys. 35, 5452 (1994); 36, 5392 (1995); C. Isham, N. Linden and S.Schreckenberg, J.Math.Phys. 35, 6360 (1994).

[15] S. Goldstein and D. N. Page, Phys.Rev.Lett. 74, 3715 (1995)

[16] J. Finkelstein, Phys.Rev. D47, 5430 (1993).

[17] H. F. Dowker and J. J. Halliwell, Phys. Rev. D46, 1580 (1992).

[18] H.F.Dowker and A.Kent, J.Stat.Phys. 82, 1575 (1996); Phys.Rev.Lett. 75, 3038 (1995).

[19] J.J.Halliwell, Phys.Rev. D63 085013 (2001).

[20] J.N.McElwaine, Phys.Rev. A53, 2021 (1996).

[21] J.B.Hartle, eprint gr-qc/9712001.

[22] J.J.Halliwell, Phys.Rev. D60, 105031 (1999).

[23] P.J.Dodd and J.J.Halliwell, e-print 0301104 (2003).

[24] I.Giardina and A.Rimini, Found.Phys. 26, 973 (1996).

[25] C.Anastopoulos, Int.J.Theor.Phys. 37, 2261 (1998).

[26] A.Caldeira and A.Leggett, Physica 121A, 587 (1983).

[27] C.Anastopoulos and J.J.Halliwell, Phys.Rev. D51, 6870 (1995).

[28] J.J.Halliwell, Phys.Rev. D46, 1610 (1992).

[29] J.J.Halliwell, Phys.Rev. D48, 2739 (1993). See also the related work, Phys.rev. D48, 4785 (1993).

[30] J.J.Halliwell, Phys.Rev. D57, 2337 (1998).

[31] L.Diosi and J.J.Halliwell, Phys.Rev.Lett. 81 2846-2849 (1998).

[32] G.Lindblad, Comm.Math.Phys. 48, 119 (1976).

[33] N.Gisin and I.C. Percival, J.Phys. A25, 5677 (1992); A26, 2233 (1993); A26, 2245 (1993); Phys. Lett. A167, 315 (1992).

[34] G.C.Ghirardi, A.Rimini and T.Weber, Phys.Rev. D34, 470 (1986); G.C.Ghirardi, P.Pearle 
amd A.Rimini, Phys.Rev. A42, 78 (1990).

[35] J.J.Halliwell and A.Zoupas, Phys.Rev. D52, 7294 (1995).

[36] L.Diósi, N.Gisin, J.Halliwell and I.C.Percival, Phys.Rev.Lett. 74, 203 (1995).

[37] T.Brun, Phys.Rev.Lett. 78, 1833 (1997); Phys.Rev. A61, 042107 (2000).

[38] J. B. Hartle, R. Laflamme and D. Marolf, Phys.Rev. D51, 7007 (1995).

[39] D. Forster, Hydrodynamic Fluctuations, Broken Symmetry and Correlation Functions (Benjamin, Reading, MA, 1975).

[40] J.J.Halliwell, Phys.Rev.Lett. 83, 2481 (1999).

[41] J.J.Halliwell, Phys.Rev. D58, 105015 (1998).

[42] C.Anastopoulos, preprint gr-qc/9805074 (1998); T.Brun and J.J.Halliwell, Phys.Rev. 54, 2899 (1996); E. Calzetta and B. L. Hu, Phys.Rev. D59, 065018 (1999), and in, Directions in General Relativity, edited by B. L. Hu and T. A. Jacobson (Cambridge University Press, Cambridge, 1993); T.Brun and J.B.Hartle, Phys.Rev. D60, 123503 (1999). See e-print quant-ph/9912037 for a review of the decoherent histories approach applied to the hydrodynamic problem.

[43] N.Yamada and S.Takagi, Prog.Theor.Phys. 85, 985 (1991); 86, 599 (1991); 87, 77 (1992); N. Yamada, Sci. Rep. Tôhoku Uni., Series 8, 12, 177 (1992); Phys.Rev. A54, 182 (1996).

[44] J. B. Hartle, Phys.Rev. D44, 3173 (1991).

[45] R.J.Micanek and J.B.Hartle, Phys.Rev. A54, 3795 (1996).

[46] J.J.Halliwell and E.Zafiris, Phys.Rev. D57, 3351 (1998).

[47] J.J.Halliwell, in Time in Quantum Mechanics, edited by J.G.Muga, R. Sala Mayato and I.L.Egususquiza (Springer, Berlin, 2001), also available as the e-print quant-ph/0101099.

[48] A.J.Leggett and A.Garg, Phys.Rev.Lett. 54, 857 (1985); J.P.Paz and G.Mahler, Phys.Rev.Lett 71, 3235 (1993).

[49] J.J.Halliwell and J.Thorwart, Phys.Rev. D64, 124018 (2001).

[50] J.J.Halliwell and J.Thorwart, Phys.Rev. D65, 104009 (2002). 